\title{
The Potential Benefits of Vonoprazan as Helicobacter pylori Infection Therapy
}

\author{
Muhammad Miftahussurur 1,2,*, Boby Pratama Putra ${ }^{3}$ and Yoshio Yamaoka ${ }^{4}$ (]) \\ 1 Division of Gastroenterology and Hepatology, Department of Internal Medicine, Faculty of Medicine, \\ Universitas Airlangga, Dr. Soetomo General Hospital, Surabaya 60286, Indonesia \\ 2 Institute of Tropical Disease, Universitas Airlangga, Surabaya 60132, Indonesia \\ 3 Faculty of Medicine, Universitas Airlangga, Surabaya 60115, Indonesia; boby.pratama-13@fk.unair.ac.id \\ 4 Department of Environmental and Preventive Medicine, Oita University Faculty of Medicine, Yufu 879-5593, \\ Japan; yyamaoka@oita-u.ac.jp \\ * Correspondence: muhammad-m@fk.unair.ac.id; Tel.: +62-315023865
}

Received: 24 August 2020; Accepted: 24 September 2020; Published: 28 September 2020

check for updates

\begin{abstract}
Helicobacter pylori infection is a severe global health problem that is closely associated with acid-related diseases and gastric malignancies. Eradicating $H$. pylori is strongly recommended for lowering peptic ulcer recurrence and preventing gastric cancer. The current approved H. pylori eradication regimen combines a proton pump inhibitor (PPI) with two antibiotics. Unfortunately, this regimen failed to meet expectations mostly due to antibiotic resistance and insufficient gastric acid suppression. Vonoprazan, a novel potassium-competitive acid blocker, showed promising results as a PPI replacement. Vonoprazan inhibits gastric acid secretion by acting as a reversible competitive inhibitor against potassium ions and forming disulfide bonds with the cysteine molecule of $\mathrm{H}^{+} / \mathrm{K}^{+}$-ATPase. Vonoprazan has superior pharmacological characteristics over PPI, such as no requirement for acid activation, stability in acidic conditions, shorter optimum acid suppression period, and resistance to cytochrome $\mathrm{P}$ (CYP)2C19 polymorphism. Several comparative randomized controlled trials and meta-analyses revealed the superiority of vonoprazan in eradicating H. pylori, notably the resistant strains. The adverse effect caused by vonoprazan is long-term acid suppression that may induce elevated gastrin serum, hypochlorhydria, and malabsorption. All vonoprazan studies have only been conducted in Japan. Further studies outside Japan are necessary for universally conclusive results.
\end{abstract}

Keywords: Helicobacter pylori; acid suppression agents; proton pump inhibitor; potassium-competitive acid blocker; vonoprazan

\section{Introduction}

Helicobacter pylori is a unique, human-specific pathogen that can be found in the human stomach in about $40-50 \%$ of the global population. H. pylori infection is a significant global health problem whose prevalence is about $44.3 \%$, from $34.7 \%$ in developed countries to $50.8 \%$ in developing countries, with a global recurrence rate of 4.3-4.6\% [1-3]. An epidemiologic meta-analysis study revealed that $H$. pylori infection is most prevalent in Africa (79.1\%), followed by Latin America (63.4\%) and Asia (54.7\%) [4]. In Indonesia, $H$. pylori infection prevalence is about $22.1 \%$, suggesting $H$. pylori infects approximately one out of five of the population [5]. H. pylori infection is significantly correlated with incidences of gastritis, gastroesophageal reflux disease, gastroduodenal ulcers, gastric mucosal-associated lymphoid tissue (MALT) lymphoma, and gastric malignancies [6-9]. The eradication of $H$. pylori is vital in reducing peptic ulcer recurrence, in the principal therapy of gastric MALT lymphoma, and in minimizing the risk of gastric cancer [10-12]. 
H. pylori elimination therapy commonly uses proton pump inhibitor (PPI)-based combination therapy for 7-14 days by combining a PPI and a minimum of two antibiotics, sometimes with the addition of bismuth. PPI takes a crucial role in $H$. pylori eradication by suppressing gastric acid secretion, hence enhancing antibiotics efficacies [13]. However, the success rate of PPI-based eradication therapy declines with antibiotics resistance emergence and inadequate acid suppression [13,14]. Increasing PPI dosage does not increase the eradication rate of PPI-based regimens [15-17]. Vonoprazan and tegoprazan are new potential gastric acid suppression agents, classified as potassium-competitive acid blockers (P-CAB), that function by $\mathrm{H}^{+} / \mathrm{K}^{+}$-ATPase inhibition $[18,19]$ (Figure 1). Japanese guidelines on the management of $H$. pylori infections recommend replacing PPI with vonoprazan in first-line and second-line H. pylori eradication therapies since first introduced in 2015, while tegoprazan has been established as treatment for gastroesophageal reflux disease (GERD) in South Korea since 2018 [20,21]. Tegoprazan showed clinical benefits in phase-III studies for erosive esophagitis patients [22] and improved both gastric-related diseases and motility defects in a canine study [23]. However, studies of tegoprazan for H. pylori eradication are still in progress. Several non-randomized control trials (RCT), $\mathrm{RCT}$, and meta-analyses reported encouraging results using vonoprazan-based therapies in eradicating H. pylori. Vonoprazan is expected to be a new candidate in H. pylori eradication regimens.

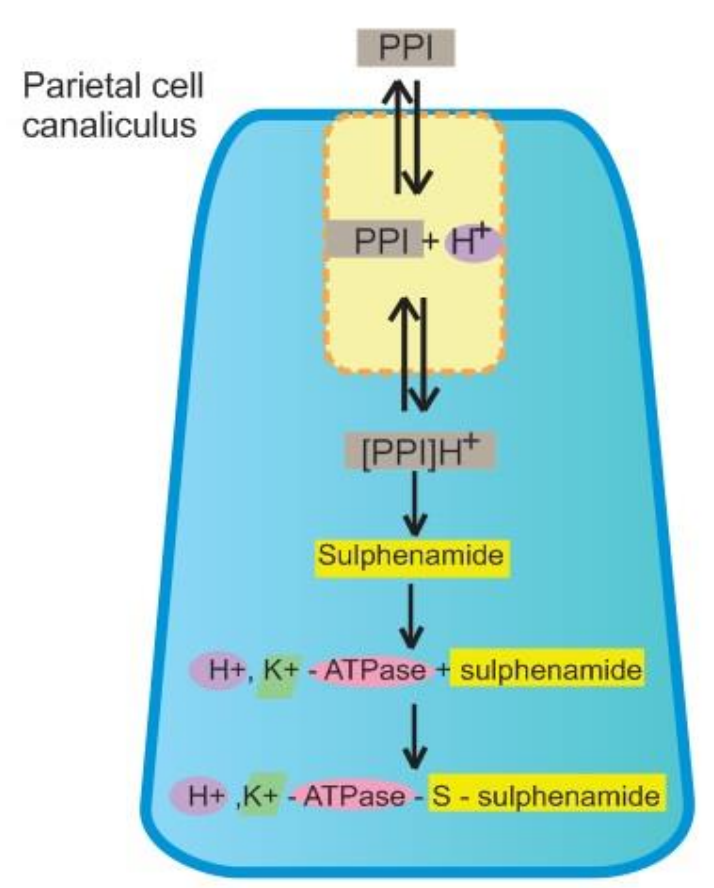

*The PPIs are inactive in their native form ${ }^{*} \mathrm{PPI}$ is unstable in acid

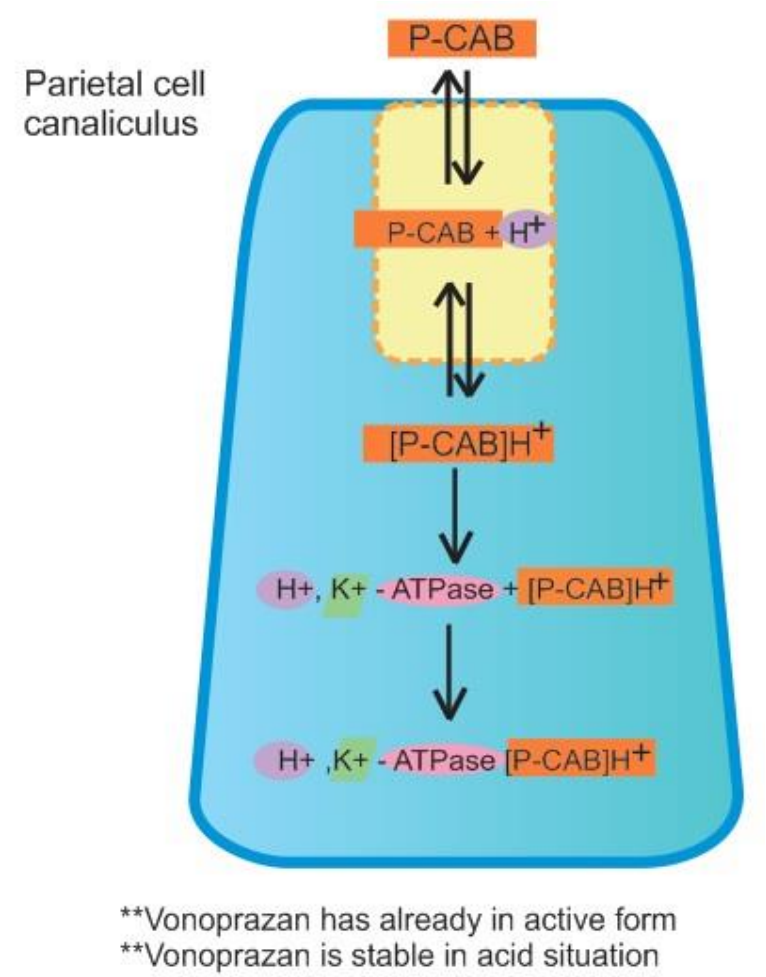

Figure 1. The mode of action of proton pump inhibitors (PPIs) and Vonoprazan against H. pylori infection. PPI enters parietal cell canaliculus in inactive form and requires acid activation. Protonated pro-drugs will convert to sulphenamide and bond covalently with cysteine groups of $\mathrm{H}^{+} / \mathrm{K}^{+}$-ATPase that cause inactivation of $\mathrm{H}^{+} / \mathrm{K}^{+}$-ATPase. Unlike PPI, potassium-competitive acid blocker (P-CAB) enters parietal cell canaliculus in active form, has stability in an acidic environment, and does not require acid activation. Protonated $\mathrm{P}-\mathrm{CAB}$ will make non-covalent bonds with $\mathrm{H}^{+} / \mathrm{K}^{+}$-ATPase, thus inactivating the $\mathrm{H}^{+} / \mathrm{K}^{+}$-ATPase with a slower dissociation rate and for longer time. ${ }^{*}$ Lacks of PPIs in gastric acid inhibition; ${ }^{* *}$ Benefits of vonoprazan in gastric acid inhibition.

We collected all relevant studies after searching comprehensively using predefined keywords through the online databases of PubMed, Web of Science, EMBASE, and The Cochrane Library. We searched all relevant articles for vonoprazan-based eradication regimens (keywords: "vonoprazan" 
OR "VPZ" OR "TAK-438" OR "Potassium-Competitive Acid Inhibitor" AND "Helicobacter pylori" OR "H. pylori") and for PPI-based eradication regimens (keywords: "proton pump inhibitor" OR "PPI" OR "omeprazole" OR "lansoprazole" OR "esomeprazole" OR "rabeprazole" AND "Helicobacter pylori" OR "H. pylori"). We first screened the title and abstract of each study and then examined full text against our inclusion criteria. We included all articles about comparative, retrospective, RCT, and meta-analysis studies of $H$. pylori eradication therapies in human populations using both regimens until April 2020. We extracted data about vonoprazan-based and PPI-based regimens with their dosages and H. pylori eradication rates. Our exclusion criteria are animal and Non-English studies.

Unsatisfactory acid-suppressing therapy outcomes prior to PPI discovery and development expedited research in obtaining new therapeutic agents. Initial studies revealed PPI has a higher efficacy than histamine-2 receptor antagonist-based therapies [24]. Table 1 reviews H. pylori eradication regimens approved by several gastroenterological societies.

Table 1. Helicobacter pylori eradication therapy regimens based on several guidelines.

\begin{tabular}{|c|c|c|c|c|}
\hline & $\begin{array}{l}\text { American College of } \\
\text { Gastroenterology [25] } \\
\text { Indonesian Society of } \\
\text { Gastroenterology [26] }\end{array}$ & $\begin{array}{c}\text { Japanese Society for } \\
\text { Helicobacter Research } \\
\text { [20] }\end{array}$ & $\begin{array}{l}\text { The Toronto Consensus } \\
{[27]}\end{array}$ & $\begin{array}{l}\text { The Maastricht V/Florence } \\
\text { Consensus Report [17] }\end{array}$ \\
\hline First Line & $\begin{array}{c}\text { If Clarithromycin-resistant } \\
\text { strains }<20 \% \\
\text { PPI * } \\
\text { Amoxicillin } \\
\text { Clarithromycin } \\
\text { If Clarithromycin-resistant } \\
\text { strains }>20 \% \\
\text { PPI * } \\
\text { Bismuth subsalicylate } \\
\text { Metronidazole } \\
\text { Tetracycline }\end{array}$ & $\begin{array}{l}\text { PPI * or Vonoprazan } \\
\text { Amoxicillin } \\
\text { Clarithromycin }\end{array}$ & $\begin{array}{c}\text { If Clarithromycin-resistant } \\
\text { strains }<15 \% \\
\text { PPI * } \\
\text { Amoxicillin/Metronidazole } \\
\text { Clarithromycin } \\
\text { If Clarithromycin-resistant } \\
\text { strains }>15 \% \\
\text { PPI * } \\
\text { Amoxicillin } \\
\text { Metronidazole } \\
\text { Clarithromycin } \\
\text { or } \\
\text { PPI * } \\
\text { Bismuth } \\
\text { Metronidazole } \\
\text { Tetracycline }\end{array}$ & $\begin{array}{c}\text { If Clarithromycin-resistant } \\
\text { strains }<15 \% \\
\text { PPI * } \\
\text { Amoxicillin } \\
\text { Clarithromycin } \\
\text { If Clarithromycin-resistant } \\
\text { strains }>15 \% \\
\text { PPI * } \\
\text { Amoxicillin } \\
\text { Metronidazole }\end{array}$ \\
\hline Second Line & $\begin{array}{c}\text { PPI* } \\
\text { Bismuth subsalicylate } \\
\text { Metronidazole } \\
\text { or } \\
\text { PPI* } \\
\text { Amoxicillin } \\
\text { Levofloxacin }\end{array}$ & $\begin{array}{c}\text { PPI * } \\
\text { Amoxicillin } \\
\text { Metronidazole }\end{array}$ & $\begin{array}{c}\text { PPI * } \\
\text { Amoxicillin } \\
\text { Levofloxacin } \\
\text { or } \\
\text { PPI * } \\
\text { Bismuth } \\
\text { Metronidazole } \\
\text { Tetracycline }\end{array}$ & $\begin{array}{c}\text { PPI * } \\
\text { Amoxicillin } \\
\text { Levofloxacin } \\
\text { or } \\
\text { PPI * } \\
\text { Bismuth } \\
\text { Metronidazole } \\
\text { Tetracycline }\end{array}$ \\
\hline Third Line & $\begin{array}{c}\text { PPI * } \\
\text { Amoxicillin } \\
\text { Levofloxacin } \\
\text { Rifabutin }\end{array}$ & $\begin{array}{c}\text { PPI }^{*} \\
\text { Amoxicillin/Metronidazole } \\
\text { Sitafloxacin }\end{array}$ & $\begin{array}{l}\text { PPI * } \\
\text { Amoxicillin } \\
\text { Rifabutin }\end{array}$ & $\begin{array}{l}\text { Regimens based on the } \\
\text { bacterial culture } \\
\text { susceptibility test }\end{array}$ \\
\hline
\end{tabular}

* PPI agents used are Omeprazole $20 \mathrm{mg}$, Lansoprazole $30 \mathrm{mg}$, Esomeprazole $40 \mathrm{mg}$, Rabeprazole $20 \mathrm{mg}$, Pantoprazole $40 \mathrm{mg}$.

Unfortunately, the clinical effectiveness of PPI-based regimens are reduced by antibiotic resistance. Failure of first-line eradication therapy is caused by the emergence of clarithromycin-resistant $H$. pylori with a failure rate of $60-70 \%[28,29]$. Otherwise, metronidazole-resistant $H$. pylori is the main cause of second-line eradication therapy, especially in Southeast Asia [30]. Resistance to levofloxacin has emerged in some countries at a resistance rate of 20-40\% [31-33]. As established previously, increasing PPI doses does not improve the eradication rate significantly. Consequently, vonoprazan was introduced as a PPI substitution candidate in all $\mathrm{H}$. pylori eradication regimens as given by the Japanese guidelines [20]. 


\section{Clinical Benefits of Vonoprazan}

\subsection{Pharmacological Aspects}

Vonoprazan is acid-stable and can act as fast-release therapy, with a maximum plasma concentration $\left(C_{\max }\right)$ that increases from 10 to $60 \mathrm{ng} / \mathrm{mL}$ in only $1.5-2 \mathrm{~h}[34,35]$. Furthermore, it has an area under curve (AUC) from no time to infinity in a dose range of 1.14-1.32 ng.h/mL and is significantly influenced by intestinal meal absorption [34-36]. Although there are no significant differences in holding time ratio at $\mathrm{pH}>4$ and time elapsed to reach $\mathrm{C}_{\max }$, vonoprazan has higher salutary $\mathrm{C}_{\max }, A U C$, and half-life compared with PPI. Its negative logarithm of acid dissociation constant $(\mathrm{pKa})>9.0$ as it is more concentrated in the secretory canaliculi of gastric parietal cells than in plasma $[36,37]$ and has higher positive charged points [38]. Its distribution depends on albumin and alpha- 1 acid glycoprotein [34].

Unlike PPI, vonoprazan does not require acid activation. It is primarily metabolized in the liver by cytochrome P450 3A4 (CYP 3A4) and metabolized partially by CYP2B6, CYP2C19, CYP2D6, and SULT2A1 [21,39]. Pharmacokinetic interaction between vonoprazan and clarithromycin is synergic because clarithromycin is a strong CYP3A4 inhibitor, which thus reduces vonoprazan metabolism [40]. Conversely, PPI is metabolized primarily by CYP2C19, which has an extensive metabolizer polymorphism that affects PPI efficacies and the prodrug activation process [21,41]. Research on acid suppression agents developed dramatically after the discovery of the crucial role of $\mathrm{H}^{+} / \mathrm{K}^{+}$-ATPase in the last stage of gastric acid secretion. PPI is a prodrug activated by acid and forms disulfide bonds with the cysteine component of $\mathrm{H}^{+} / \mathrm{K}^{+}$-ATPase [37,42]. PPI reaches maximum acid stability after $3-5$ days of treatment $[43,44]$.

The inability of PPI to create a gastric base environment has led researchers to investigate alternative acid-suppressing agents. One possible alternative mechanism is the reduction of potassium ion concentration to limit $\mathrm{H}^{+} / \mathrm{K}^{+}$-ATPase efficacy. P-CAB agents, such as vonoprazan, act as a reversible competitive inhibitors against potassium ions by binding with $\mathrm{H}^{+} / \mathrm{K}^{+}$-ATPase $[45,46]$. Vonoprazan is stable in the acidic gastric secretory canaliculi environment and binds non-covalently to $\mathrm{H}^{+} / \mathrm{K}^{+}$-ATPase [47]. Vonoprazan dissociates gradually and represses $\mathrm{H}^{+} / \mathrm{K}^{+}$-ATPase formation for a sustained period, consequently increasing gastric $\mathrm{pH}$ up to $\mathrm{pH} 7$ in approximately in $4 \mathrm{~h}$ [48]. The differences between the pharmacokinetics and pharmacodynamics of PPI and Vonoprazan are shown in Table $2[44,46]$.

Vonoprazan has potential to substitute PPI in GERD and gastroduodenal ulcer management. Standard therapy for these diseases is PPI, yet the outcomes are unsatisfactory. Substituting PPI for vonoprazan in erosive esophagitis can relieve symptoms quickly and significantly. A meta-analysis proved the superiority of vonoprazan against PPI in GERD management and subgroup analysis noted that vonoprazan significantly has higher efficacy in treating erosive esophagitis [49], especially in CYP2C19 EM patients with an efficacy rate of $90.0 \%$, compared with $79.3 \%$ for PPI [50]. Vonoprazan is also superior against lansoprazole in treating peptic ulcer, with recurrence rates at $3.3 \%$ vs. $5.5 \%$, respectively, as confirmed by endoscopy examination [51]. An RCT study confirmed the high efficacy of vonoprazan in peptic ulcer treatment, at $93.5 \%$ compared with lansoprazole at $93.8 \%$. However, the study could not establish its efficacy in duodenal ulcer treatment due to dropped out patients and recurring ulcers [52]. Vonoprazan also has comparable efficacy with lansoprazole in reducing peptic ulcer recurrence incidence in patients taking low-dose aspirin [53]. Furthermore, a meta-analysis showed that patients who received vonoprazan for peptic ulcer related to endoscopic gastric submucosal resection have statistically significantly higher healing rates compared with those who received PPI (pooled odds ratio (OR) 2.27, 95\% CI 1.38-3.73, heterogeneity $\left(\mathrm{I}^{2}\right)=0 \%, p=0.001$ ) [54]. 
Table 2. Pharmacological comparisons between PPI and Vonoprazan.

\begin{tabular}{|c|c|c|c|}
\hline \multirow{2}{*}{ Parameter } & \multicolumn{2}{|c|}{ PPI } & \multirow{2}{*}{ Vonoprazan } \\
\hline & First Generation PPI & Second Generation PPI & \\
\hline Acid activation & \multicolumn{2}{|r|}{ Yes } & No \\
\hline Active drug & \multicolumn{2}{|c|}{ No } & Yes \\
\hline Acid Stability & \multicolumn{2}{|c|}{ No } & Yes \\
\hline Main P450 metabolizer & \multicolumn{2}{|c|}{ CYP2C19 } & CYP3A4 \\
\hline Meal's influence & \multicolumn{2}{|c|}{ Yes } & No \\
\hline Mechanism of Action & \multicolumn{2}{|c|}{ Covalent bond to gastric proton pump } & $\begin{array}{l}\text { Potassium ion competitive } \\
\text { reversible inhibitor to gastric } \\
\text { proton pump }\end{array}$ \\
\hline $\begin{array}{l}\text { Days required for } \\
\text { reaching maximal acid } \\
\text { suppression }\end{array}$ & \multicolumn{2}{|c|}{$3-5$} & 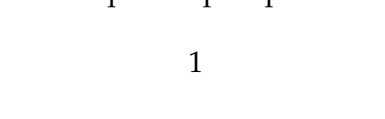 \\
\hline \multirow{2}{*}{$\mathrm{pH}>4$ holding time $(\%)$} & OMZ 30.4 & ESO 43.1 & $10 \mathrm{mg} 38.4-43.1$ \\
\hline & LPZ 39.1 & RPZ 42.8 & $20 \mathrm{mg} 62.7-63.3$ \\
\hline \multirow{2}{*}{$\begin{array}{l}\text { Time Needed to Reach } \\
\text { Maximum Plasma } \\
\text { Concentration (h) }\end{array}$} & OMZ 1-4 & ESO 1-3.5 & $10 \mathrm{mg} 1.75$ \\
\hline & LPZ 1.2-2.1 & RPZ 1.14 & $20 \mathrm{mg} 1.50$ \\
\hline \multirow{2}{*}{ Half-life (h) } & OMZ 0.5-1.2 & ESO 1.3-1.6 & $10 \mathrm{mg} 6.95 \pm 1.03$ \\
\hline & LPZ 0.9-2.1 & RPZ 0.6-1.4 & $20 \mathrm{mg} 6.85 \pm 0.80$ \\
\hline \multirow{2}{*}{$\mathrm{C}_{\max }(\mu \mathrm{mol} / \mathrm{L})$} & OMZ 0.23-23.2 & ESO 2.1-2.4 & $10 \mathrm{mg} 9.7 \pm 2.1 \mu \mathrm{g} / \mathrm{L}$ \\
\hline & LPZ 1.62-3.25 & RPZ 1.14 & $20 \mathrm{mg} 25.0 \pm 5.6 \mu \mathrm{g} / \mathrm{L}$ \\
\hline \multirow{2}{*}{ AUC $(\mu \mathrm{mol} . \mathrm{h} / \mathrm{L})$} & OMZ 0.58-3.47 & ESO 4.2 & $10 \mathrm{mg} 60.1 \pm 9.0 \mu \mathrm{g} . \mathrm{h} / \mathrm{L}$ \\
\hline & LPZ 4.60-13.5 & RPZ 2.22 & $20 \mathrm{mg} 160.3 \pm 38.6 \mu \mathrm{g} . \mathrm{h} / \mathrm{L}$ \\
\hline
\end{tabular}

AUC: Area Under Curve; $C_{\max }$ : Maximum Plasma Concentration; CYP: Cytochrome P450 OMZ: Omeprazole 20 mg; LPZ: Lansoprazole $30 \mathrm{mg}$; ESO: Esomeprazole 40 mg; RPZ: Rabeprazole $20 \mathrm{mg}$.

\subsection{Vonoprazan and H. pylori Eradication}

H. pylori eradication is essential for preventing and intervening in long-term complications. Determinants influencing the eradication rate of $H$. pylori eradication therapy include antibiotic resistance, acid suppression adequacy, virulence factors (cagA, vacA, dupA), and the environment [55-58]. PPI-based H. pylori eradication therapy regimens have been ineffective, and the efficacy of doubling the PPI dose has little evidence and weak recommendations [17]. In addition, the CYP2C19 extensive metabolizer polymorphism diminishes PPI ability in suppressing gastric acid.

Vonoprazan is a strong candidate replacement for PPI in H. pylori eradication regimens. Its pharmacological advantages include no requirement of acid activation, stability in an acidic environment, and a longer half-life [55]. Standardized first-line H. pylori eradication therapy is PPI, clarithromycin, and amoxicillin. RCT and non-RCT studies have revealed that vonoprazan-based eradication regimens have a higher eradication rate than PPI-based regimens (Table 3). Our previous meta-analysis of five clarithromycin-sensitive $H$. pylori RCT studies revealed no statistically significant differences between the eradication rates of first-generation PPI-based (pooled risk ratio (RR) 1.01, $95 \%$ CI $0.98-1.04, \mathrm{I}^{2}=61 \%, p=0.04$ ) and vonoprazan-based regimens (pooled RR $0.84,95 \%$ CI $\left.0.57-1.25, \mathrm{I}^{2}=0 \%, p=0.39\right)$. However, we found significant differences between the eradication rates of second-generation PPI-based (pooled RR 1.25, 95\% CI 1.15-1.37, $\mathrm{I}^{2}=82 \%, p<0.00001$ ) and vonoprazan-based regimens (pooled RR 0.31, 95\% CI 0.23-0.42, $\mathrm{I}^{2}=50 \%, p<0.00001$ ), as well as between all PPI generation-based (pooled RR 1.11,95\% CI 1.07-1.16, $\mathrm{I}^{2}=98 \%, p<0.00001$ ) and vonoprazan-based regimens combined (pooled RR $0.43,95 \%$ CI $0.34-0.55, \mathrm{I}^{2}=81 \%, p<0.00001$ ) [15]. Several studies on vonoprazan-based regimens showed higher eradication rates against clarithromycin-resistant $H$. pylori (Table 4). Furthermore, a meta-analysis concluded that vonoprazan-based regimens have superiority in eradicating clarithromycin-resistant $H$. pylori (pooled eradication rates $82 \%$ and $40 \%$, pooled OR $6.83,95 \%$ CI 3.63-12.86, $\mathrm{I}^{2}=0 \%, p<0.0001$ ) [59]. 
Table 3. Review of Comparative Studies First-line H. pylori Eradication Therapy.

\begin{tabular}{|c|c|c|c|c|}
\hline \multirow{2}{*}{ Study } & \multicolumn{2}{|c|}{ VPZ-Based Regimen } & \multicolumn{2}{|c|}{ PPI-Based Regimen } \\
\hline & Regimen & Eradication Rate & Regimen & Eradication Rate \\
\hline \multicolumn{5}{|l|}{ RCT } \\
\hline Murakami et al., 2016 [60] & $\begin{array}{c}\text { VPZ: } 20 \mathrm{mg} \text { bid } \\
\text { AMX: } 750 \mathrm{mg} \text { bid } \\
\text { CLR: } 200 \text { or } 400 \mathrm{mg} \text { bid }\end{array}$ & $90.9 \%$ & $\begin{array}{c}\text { LPZ: } 30 \mathrm{mg} \text { bid } \\
\text { AMX: } 750 \mathrm{mg} \text { bid } \\
\text { CLR: } 200 \text { or } 400 \mathrm{mg} \text { bid }\end{array}$ & $75.1 \%$ \\
\hline Maruyama et al., 2017 [61] & $\begin{array}{c}\text { VPZ: } 20 \mathrm{mg} \text { bid } \\
\text { AMX: } 750 \mathrm{mg} \text { bid } \\
\text { CLR: } 200 \text { or } 400 \mathrm{mg} \text { bid }\end{array}$ & $95.8 \%$ & $\begin{array}{l}\text { LPZ: } 30 \mathrm{mg} \text { bid or } \\
\text { RPZ: } 20 \mathrm{mg} \text { bid } \\
\text { AMX: } 750 \mathrm{mg} \text { bid } \\
\text { CLR: } 200 \text { or } 400 \mathrm{mg} \text { bid }\end{array}$ & $69.6 \%$ \\
\hline Sue et al., 2017 [62] & $\begin{array}{c}\text { VPZ: } 20 \mathrm{mg} \text { bid } \\
\text { AMX: } 750 \mathrm{mg} \text { bid } \\
\text { CLR: } 200 \text { or } 400 \mathrm{mg} \text { bid }\end{array}$ & $87.3 \%$ & $\begin{array}{l}\text { LPZ: } 30 \mathrm{mg} \text { bid, } \\
\text { RPZ: } 10 \mathrm{mg} \text { bid or } \\
\text { ESO: } 20 \mathrm{mg} \text { bid } \\
\text { AMX: } 750 \mathrm{mg} \text { bid } \\
\text { CLR: } 200 \text { or } 400 \mathrm{mg} \text { bid }\end{array}$ & $76.5 \%$ \\
\hline Ozaki et al., 2018 [63] & $\begin{array}{c}\text { VPZ: } 20 \mathrm{mg} \text { bid } \\
\text { AMX: } 750 \mathrm{mg} \text { bid } \\
\text { CLR: } 200 \text { or } 400 \mathrm{mg} \text { bid }\end{array}$ & $90.9 \%$ & $\begin{array}{l}\text { RPZ: } 10 \mathrm{mg} \text { bid or } \\
\text { ESO: } 20 \mathrm{mg} \text { bid } \\
\text { AMX: } 750 \mathrm{mg} \text { bid } \\
\text { CLR: } 200 \text { or } 400 \mathrm{mg} \text { bid }\end{array}$ & $72.8 \%$ \\
\hline \multicolumn{5}{|l|}{ Non-RCT } \\
\hline Suzuki et al., 2016 [64] & $\begin{array}{c}\text { VPZ: } 20 \mathrm{mg} \text { bid } \\
\text { AMX: } 750 \mathrm{mg} \text { bid } \\
\text { CLR: } 200 \text { or } 400 \mathrm{mg} \text { bid }\end{array}$ & $89.0 \%$ & $\begin{array}{l}\text { LPZ: } 30 \mathrm{mg} \text { bid or } \\
\text { RPZ: } 20 \mathrm{mg} \text { bid } \\
\text { AMX: } 750 \mathrm{mg} \text { bid } \\
\text { CLR: } 200 \mathrm{mg} \text { bid }\end{array}$ & $74.2 \%$ \\
\hline Shinozaki et al., 2016 [65] & $\begin{array}{c}\text { VPZ: } 20 \mathrm{mg} \text { bid } \\
\text { AMX: } 750 \mathrm{mg} \text { bid } \\
\text { CLR: } 200 \text { or } 400 \mathrm{mg} \text { bid }\end{array}$ & $82.9 \%$ & $\begin{array}{l}\text { LPZ: } 30 \mathrm{mg} \text { bid, } \\
\text { RPZ: } 10 \mathrm{mg} \text { bid or } \\
\text { ESO: } 20 \mathrm{mg} \text { bid } \\
\text { AMX: } 750 \mathrm{mg} \text { bid } \\
\text { CLR: } 200 \mathrm{mg} \text { bid }\end{array}$ & $73.9 \%$ \\
\hline Shichijo et al., 2016 [66] & $\begin{array}{c}\text { VPZ: } 20 \mathrm{mg} \text { bid } \\
\text { AMX: } 750 \mathrm{mg} \text { bid } \\
\text { CLR: } 200 \text { or } 400 \mathrm{mg} \text { bid }\end{array}$ & $87.2 \%$ & $\begin{array}{c}\text { LPZ: } 30 \mathrm{mg} \text { bid, } \\
\text { RPZ: } 10 \mathrm{mg} \text { bid or } \\
\text { ESO: } 20 \mathrm{mg} \text { bid } \\
\text { AMX: } 750 \mathrm{mg} \text { bid } \\
\text { CLR: } 200 \text { or } 400 \mathrm{mg} \text { bid }\end{array}$ & $72.4 \%$ \\
\hline Noda et al., 2016 [67] & $\begin{array}{l}\text { VPZ: } 20 \mathrm{mg} \text { bid } \\
\text { AMX: } 750 \mathrm{mg} \text { bid } \\
\text { CLR: } 400 \mathrm{mg} \text { bid }\end{array}$ & $89.7 \%$ & $\begin{array}{l}\text { OMZ: } 20 \mathrm{mg} \text { bid, } \\
\text { LPZ: } 30 \mathrm{mg} \text { bid, } \\
\text { RPZ: } 10 \mathrm{mg} \text { bid or } \\
\text { ESO: } 20 \mathrm{mg} \text { bid } \\
\text { AMX: } 750 \mathrm{mg} \text { bid } \\
\text { CLR: } 200 \text { or } 400 \mathrm{mg} \text { bid }\end{array}$ & $73.9 \%$ \\
\hline Matsumoto et al., 2016 [68] & $\begin{array}{l}\text { VPZ: } 20 \mathrm{mg} \text { bid } \\
\text { AMX: } 750 \mathrm{mg} \text { bid } \\
\text { CLR: } 200 \mathrm{mg} \text { bid }\end{array}$ & $89.6 \%$ & $\begin{array}{l}\text { LPZ: } 30 \mathrm{mg} \text { bid, } \\
\text { RPZ: } 10 \mathrm{mg} \text { bid or } \\
\text { ESO: } 20 \mathrm{mg} \text { bid } \\
\text { AMX: } 750 \mathrm{mg} \text { bid } \\
\text { CLR: } 200 \text { or } 400 \mathrm{mg} \text { bid }\end{array}$ & $71.9 \%$ \\
\hline Yamada et al., 2016 [69] & $\begin{array}{l}\text { VPZ: } 20 \mathrm{mg} \text { bid } \\
\text { AMX: } 750 \mathrm{mg} \text { bid } \\
\text { CLR: } 200 \mathrm{mg} \text { bid }\end{array}$ & $85.7 \%$ & $\begin{array}{l}\text { LPZ: } 30 \mathrm{mg} \text { bid, } \\
\text { RPZ: } 10 \mathrm{mg} \text { bid or } \\
\text { ESO: } 20 \mathrm{mg} \text { bid } \\
\text { AMX: } 750 \mathrm{mg} \text { bid } \\
\text { CLR: } 200 \mathrm{mg} \text { bid }\end{array}$ & $73.2 \%$ \\
\hline Tsujimae et al., 2016 [70] & $\begin{array}{l}\text { VPZ: } 20 \mathrm{mg} \text { bid } \\
\text { AMX: } 750 \mathrm{mg} \text { bid } \\
\text { CLR: } 200 \mathrm{mg} \text { bid }\end{array}$ & $84.6 \%$ & $\begin{array}{l}\text { ESO: } 20 \mathrm{mg} \text { bid } \\
\text { AMX: } 750 \mathrm{mg} \text { bid } \\
\text { CLR: } 200 \mathrm{mg} \text { bid }\end{array}$ & $79.1 \%$ \\
\hline
\end{tabular}


Table 3. Cont.

\begin{tabular}{|c|c|c|c|c|}
\hline \multirow{2}{*}{ Study } & \multicolumn{2}{|c|}{ VPZ-Based Regimen } & \multicolumn{2}{|c|}{ PPI-Based Regimen } \\
\hline & Regimen & Eradication Rate & Regimen & Eradication Rate \\
\hline Kajihara et al., 2016 [71] & $\begin{array}{l}\text { VPZ: } 20 \mathrm{mg} \text { bid } \\
\text { AMX: } 750 \mathrm{mg} \text { bid } \\
\text { CLR: } 400 \mathrm{mg} \text { bid }\end{array}$ & $94.6 \%$ & $\begin{array}{c}\text { RPZ: } 10 \mathrm{mg} \text { bid } \\
\text { AMX: } 750 \mathrm{mg} \text { bid } \\
\text { CLR: } 200 \text { or } 400 \mathrm{mg} \text { bid }\end{array}$ & $86.7 \%$ \\
\hline Sakurai et al., 2017 [72] & $\begin{array}{l}\text { VPZ: } 20 \mathrm{mg} \text { bid } \\
\text { AMX: } 750 \mathrm{mg} \text { bid } \\
\text { CLR: } 200 \mathrm{mg} \text { bid }\end{array}$ & $87.9 \%$ & $\begin{array}{l}\text { LPZ: } 30 \mathrm{mg} \text { bid, } \\
\text { RPZ: } 10 \mathrm{mg} \text { bid or } \\
\text { ESO: } 20 \mathrm{mg} \text { bid } \\
\text { AMX: } 750 \mathrm{mg} \text { bid } \\
\text { CLR: } 200 \mathrm{mg} \text { bid }\end{array}$ & $66.9 \%$ \\
\hline Sue et al., 2017 [73] & $\begin{array}{c}\text { VPZ: } 20 \mathrm{mg} \text { bid } \\
\text { AMX: } 750 \mathrm{mg} \text { bid } \\
\text { CLR: } 200 \text { or } 400 \mathrm{mg} \text { bid }\end{array}$ & $84.9 \%$ & $\begin{array}{l}\text { OMZ: } 20 \mathrm{mg} \text { bid, } \\
\text { LPZ: } 30 \mathrm{mg} \text { bid, } \\
\text { RPZ: } 10 \mathrm{mg} \text { bid or } \\
\text { ESO: } 20 \mathrm{mg} \text { bid } \\
\text { AMX: } 750 \mathrm{mg} \text { bid } \\
\text { CLR: } 200 \text { or } 400 \mathrm{mg} \text { bid }\end{array}$ & $78.8 \%$ \\
\hline Nishizawa et al., 2017 [74] & $\begin{array}{c}\text { VPZ: } 20 \mathrm{mg} \text { bid } \\
\text { AMX: } 750 \mathrm{mg} \text { bid } \\
\text { CLR: } 200 \text { or } 400 \mathrm{mg} \text { bid }\end{array}$ & $62.3 \%$ & $\begin{array}{c}\text { LPZ: } 30 \mathrm{mg} \text { bid or } \\
\text { RPZ: } 10 \mathrm{mg} \text { bid } \\
\text { AMX: } 750 \mathrm{mg} \text { bid } \\
\text { CLR: } 200 \text { or } 400 \mathrm{mg} \text { bid }\end{array}$ & $47.1 \%$ \\
\hline Tanabe et al., 2018 [75] & $\begin{array}{c}\text { VPZ: } 20 \mathrm{mg} \text { bid } \\
\text { AMX: } 750 \mathrm{mg} \text { bid } \\
\text { CLR: } 200 \text { or } 400 \mathrm{mg} \text { bid }\end{array}$ & $91.5 \%$ & $\begin{array}{l}\text { LPZ: } 30 \mathrm{mg} \text { bid, } \\
\text { RPZ: } 10 \mathrm{mg} \text { bid or } \\
\text { ESO: } 20 \mathrm{mg} \text { bid } \\
\text { AMX: } 750 \mathrm{mg} \text { bid } \\
\text { CLR: } 200 \mathrm{mg} \text { bid }\end{array}$ & $79.4 \%$ \\
\hline
\end{tabular}

AMX: Amoxicillin, CLR: Clarithromycin, ESO: Esomeprazole, LPZ: Lansoprazole, OMZ: Omeprazole, RPZ: Rabeprazole, VPZ: Vonoprazan.

Table 4. Review of comparative studies of first-line clarithromycin-resistant $H$. pylori eradication therapies.

\begin{tabular}{|c|c|c|c|c|}
\hline \multirow{2}{*}{ Study } & \multicolumn{2}{|c|}{ VPZ-Based Regimen } & \multicolumn{2}{|c|}{ PPI-Based Regimen } \\
\hline & Regimen & Eradication Rate & Regimen & Eradication Rate \\
\hline \multicolumn{5}{|l|}{$\mathrm{RCT}$} \\
\hline Murakami et al., 2016 [60] & $\begin{array}{c}\text { VPZ: } 20 \mathrm{mg} \text { bid } \\
\text { AMX: } 750 \mathrm{mg} \text { bid } \\
\text { CLR: } 200 \text { or } 400 \mathrm{mg} \text { bid }\end{array}$ & $82.0 \%$ & $\begin{array}{c}\text { LPZ: } 30 \mathrm{mg} \text { bid } \\
\text { AMX: } 750 \mathrm{mg} \text { bid } \\
\text { CLR: } 200 \text { or } 400 \mathrm{mg} \text { bid }\end{array}$ & $40.0 \%$ \\
\hline \multicolumn{5}{|l|}{ Non-RCT } \\
\hline Noda et al., 2016 [67] & $\begin{array}{l}\text { VPZ: } 20 \mathrm{mg} \text { bid } \\
\text { AMX: } 750 \mathrm{mg} \text { bid } \\
\text { CLR: } 400 \mathrm{mg} \text { bid }\end{array}$ & $87.5 \%$ & $\begin{array}{l}\text { OMZ: } 20 \mathrm{mg} \text { bid, } \\
\text { LPZ: } 30 \mathrm{mg} \text { bid, } \\
\text { RPZ: } 10 \mathrm{mg} \text { bid or } \\
\text { ESO: } 20 \mathrm{mg} \text { bid } \\
\text { AMX: } 750 \mathrm{mg} \text { bid } \\
\text { CLR: } 200 \text { or } 400 \mathrm{mg} \text { bid }\end{array}$ & $53.8 \%$ \\
\hline Matsumoto et al., 2016 [68] & $\begin{array}{l}\text { VPZ: } 20 \mathrm{mg} \text { bid } \\
\text { AMX: } 750 \mathrm{mg} \text { bid } \\
\text { CLR: } 200 \mathrm{mg} \text { bid }\end{array}$ & $76.1 \%$ & $\begin{array}{c}\text { LPZ: } 30 \mathrm{mg} \text { bid, } \\
\text { RPZ: } 10 \mathrm{mg} \text { bid or } \\
\text { ESO: } 20 \mathrm{mg} \text { bid } \\
\text { AMX: } 750 \mathrm{mg} \text { bid } \\
\text { CLR: } 200 \text { or } 400 \mathrm{mg} \text { bid }\end{array}$ & $40.2 \%$ \\
\hline
\end{tabular}

AMX: Amoxicillin, CLR: Clarithromycin, ESO: Esomeprazole, LPZ: Lansoprazole, OMZ: Omeprazole, RPZ: Rabeprazole, VPZ: Vonoprazan.

Standard second-line H. pylori eradication therapy, which is used after the failure of first-line therapy, consists of PPI, amoxicillin, and metronidazole. We did not find any RCT studies comparing the outcomes of vonoprazan-based and PPI-based second-line H. pylori eradication therapies (Table 5). Shinozaki et al. conducted a meta-analysis of non-RCT studies and concluded that vonoprazan-based second-line eradication regimens are statistically significant in eradicating H. pylori (pooled OR 1.51, $95 \%$ CI $1.27-1.81, \mathrm{I}^{2}=0 \%, p<0.00001$ ) [76]. 
Table 5. Review of comparative studies of second-line H. pylori eradication therapies.

\begin{tabular}{|c|c|c|c|c|}
\hline \multirow{2}{*}{ Study } & \multicolumn{2}{|c|}{ VPZ-Based Regimen } & \multicolumn{2}{|c|}{ PPI-Based Regimen } \\
\hline & Regimen & Eradication Rate & Regimen & Eradication Rate \\
\hline Yamada et al., 2016 [69] & $\begin{array}{l}\text { VPZ: } 20 \mathrm{mg} \text { bid } \\
\text { AMX: } 750 \mathrm{mg} \text { bid } \\
\text { MNZ: } 250 \mathrm{mg} \text { bid }\end{array}$ & $89.6 \%$ & $\begin{array}{l}\text { LPZ: } 30 \mathrm{mg} \text { bid, } \\
\text { RPZ: } 10 \mathrm{mg} \text { bid or } \\
\text { ESO: } 20 \mathrm{mg} \text { bid } \\
\text { AMX: } 750 \mathrm{mg} \text { bid } \\
\text { MNZ: } 250 \mathrm{mg} \text { bid }\end{array}$ & $89.9 \%$ \\
\hline Tsujimae et al., 2016 [70] & $\begin{array}{l}\text { VPZ: } 20 \mathrm{mg} \text { bid } \\
\text { AMX: } 750 \mathrm{mg} \text { bid } \\
\text { MNZ: } 250 \mathrm{mg} \text { bid }\end{array}$ & $89.1 \%$ & $\begin{array}{l}\text { ESO: } 20 \mathrm{mg} \text { bid } \\
\text { AMX: } 750 \mathrm{mg} \text { bid } \\
\text { MNZ: } 250 \mathrm{mg} \text { bid }\end{array}$ & $83.3 \%$ \\
\hline Sakurai et al., 2017 [72] & $\begin{array}{l}\text { VPZ: } 20 \mathrm{mg} \text { bid } \\
\text { AMX: } 750 \mathrm{mg} \text { bid } \\
\text { MNZ: } 250 \mathrm{mg} \text { bid }\end{array}$ & $96.1 \%$ & $\begin{array}{l}\text { LPZ: } 30 \mathrm{mg} \text { bid, } \\
\text { RPZ: } 10 \mathrm{mg} \text { bid or } \\
\text { ESO: } 20 \mathrm{mg} \text { bid } \\
\text { AMX: } 750 \mathrm{mg} \text { bid } \\
\text { MNZ: } 250 \mathrm{mg} \text { bid }\end{array}$ & $89.7 \%$ \\
\hline Sue et al., 2017 [73] & $\begin{array}{l}\text { VPZ: } 20 \mathrm{mg} \text { bid } \\
\text { AMX: } 750 \mathrm{mg} \text { bid } \\
\text { MNZ: } 250 \mathrm{mg} \text { bid }\end{array}$ & $80.5 \%$ & $\begin{array}{l}\text { LPZ: } 30 \mathrm{mg} \text { bid, } \\
\text { RPZ: } 10 \mathrm{mg} \text { bid or } \\
\text { ESO: } 20 \mathrm{mg} \text { bid } \\
\text { AMX: } 750 \mathrm{mg} \text { bid } \\
\text { MNZ: } 250 \mathrm{mg} \text { bid }\end{array}$ & $81.5 \%$ \\
\hline Nishizawa et al., 2017 [77] & $\begin{array}{l}\text { VPZ: } 20 \mathrm{mg} \text { bid } \\
\text { AMX: } 750 \mathrm{mg} \text { bid } \\
\text { MNZ: } 250 \mathrm{mg} \text { bid }\end{array}$ & $71.8 \%$ & $\begin{array}{l}\text { LPZ: } 30 \mathrm{mg} \text { bid or } \\
\text { RPZ: } 10 \mathrm{mg} \text { bid } \\
\text { AMX: } 750 \mathrm{mg} \text { bid } \\
\text { MNZ: } 250 \mathrm{mg} \text { bid }\end{array}$ & $73.7 \%$ \\
\hline
\end{tabular}

AMX: Amoxicillin, CLR: Clarithromycin, ESO: Esomeprazole, LPZ: Lansoprazole, MNZ: Metronidazole, RPZ: Rabeprazole, VPZ: Vonoprazan.

Third-line H. pylori eradication regimens combine PPI or vonoprazan with amoxicillin and sitafloxacin. A study revealed that the third-line vonoprazan-based regimen has a higher H. pylori eradication rate than the PPI-based regimen (75.8\% vs. 53.3\%) [78]. Another study also showed the vonoprazan-based regimen has a higher eradication rate against sitafloxacin-resistant $H$. pylori than esomeprazole-based regimens (91.7\% vs. $71.2 \%$ ) [79]. Studies on third-line H. pylori eradication therapies are limited because third-line therapies are not covered under the universal Japanese health insurance [80].

The main limitation in this review is that all studies were conducted in Japan, which thus raises the question on the efficacy rates of vonoprazan outside Japan. For one, the Japanese population tends to have a higher holding time ratio at $\mathrm{pH}>4$ than the UK population [35,36]. For another, every region has a different antibiotic resistance mapping. For example, Japan has a high clarithromycin resistance rate $(>30 \%)$ but a low metronidazole resistance rate $(<5 \%)$ [81]. A contradictory study conducted in Indonesia revealed that H. pylori in the country has low clarithromycin resistance (9.1\%) but high metronidazole and levofloxacin resistances at the rates of $46.7 \%$ and $31.2 \%$, respectively [33].

A high incidence of $H$. pylori with poly-antimicrobial resistances compels research into alternative $H$. pylori eradication therapies. Previously, we investigated alternative therapies against metronidazole-resistant and levofloxacin-resistant H. pylori strains in Indonesia, Bangladesh, and Bhutan in vitro and found that furazolidones, rifaximin, rifabutin, garenoxacin, and sitafloxacin are effective in eradicating $H$. pylori [33,82]. Alternative therapy using herbal medicine such as the Indian plant Bombax ceiba, or a propolis (Trigona sp.) ethanol extract can inhibit the growth of metronidazole-resistant and levofloxacin-resistant $H$. pylori in vitro $[83,84]$.

\subsection{Safety and Adverse Events}

Since the discovery of $\mathrm{P}-\mathrm{CAB}$, the most recognized complication is hepatotoxicity, although no serious adverse effects have been observed $[42,44]$. Unlike the previous P-CAB group, which is a 
derivative of an imidazole-pyridine compound, vonoprazan is a pyridine-derivative compound, which has a lower hepatotoxicity risk $[21,85]$. Nevertheless, some previous studies did not encounter any significant difference between transaminase increases in patients receiving vonoprazan and PPI [34].

The acid inhibition rate of vonoprazan is better than that of PPI. Consequently, the increase in gastrin serum in patients receiving vonoprazan therapy is higher than in patients receiving PPI therapy $[16,50]$. Hypergastrinemia can trigger gastric enterochromaffin cell hyperplasia and increase the risk of gastric endocrine tumors [86,87]. Hypochlorhydria precipitated by acid inhibition can alter the gut microbiome, increasing the risk of developing antibiotic-associated diarrhea caused by Clostridium difficile and spontaneous bacterial peritonitis [88,89]. Excessive acid suppression can also cause malabsorption, resulting in the onset of iron deficiency anemia, megaloblastic anemia, hypomagnesia, and hypocalcemia [46,90]. Additional side effects that can emerge include interstitial nephritis, pneumonia, dementia, chronic kidney disease, and ischemic heart disease [91-93].

\section{Conclusions}

Vonoprazan is a promising alternative to PPI in the treatment of gastroduodenal diseases, mainly in H. pylori eradication therapy, given its superior pharmacological and clinical indications to PPI. However, further clinical studies on vonoprazan are required to confirm its efficacies, especially outside Japan, in order to establish universally conclusive results.

Author Contributions: M.M. and B.P.P. searched articles independently, extracted data, drafted, and approved the final manuscript. Y.Y. drafted and approved the final manuscript. All authors have read and agreed to the published version of the manuscript.

Funding: This research was funded by Hibah Riset Mandat 2020 Grant from Universitas Airlangga number 377/UN3.14/PT/2020 and the article processing charge was funded by Faculty of Medicine, Universitas Airlangga, Surabaya, Indonesia.

Conflicts of Interest: The authors declare no conflict of interest.

\section{References}

1. Hu, Y.; Wan, J.H.; Li, X.Y.; Zhu, Y.; Graham, D.Y.; Lu, N.H. Systematic review with meta-analysis: The global recurrence rate of Helicobacter pylori. Aliment. Pharmacol. Ther. 2017, 46, 773-779. [CrossRef] [PubMed]

2. Xue, Y.; Zhou, L.Y.; Lu, H.P.; Liu, J.Z.; Guo, L.S. Recurrence of Helicobacter pylori infection: Incidence and influential factors. Chin. Med. J. (Engl.) 2019, 132, 765-771. [CrossRef] [PubMed]

3. Sjomina, O.; Pavlova, J.; Niv, Y.; Leja, M. Epidemiology of Helicobacter pylori infection. Helicobacter 2018, 23, 6-11. [CrossRef] [PubMed]

4. Hooi, J.K.Y.; Lai, W.Y.; Ng, W.K.; Suen, M.M.Y.; Underwood, F.E.; Tanyingoh, D.; Malfertheiner, P.; Graham, D.Y.; Wong, V.W.S.; Wu, J.C.; et al. Global Prevalence of Helicobacter pylori Infection: Systematic Review and Meta-Analysis. Gastroenterology 2017, 153, 420-429. [CrossRef]

5. Syam, A.F.; Miftahussurur, M.; Makmun, D.; Nusi, I.A.; Zain, L.H.; Zulkhairi; Akil, F.; Uswan, W.B.; Simanjuntak, D.; Uchida, T.; et al. Risk factors and prevalence of Helicobacter pylori in five largest islands of Indonesia: A preliminary study. PLoS ONE 2015, 10, e0140186. [CrossRef]

6. Abadi, A.T.B.; Ierardi, E. Vonoprazan and Helicobacter pylori treatment: A lesson from Japan or a limited geographic phenomenon? Front. Pharmacol. 2019, 10,1-6. [CrossRef]

7. Lyu, Q.J.; Pu, Q.H.; Zhong, X.F.; Zhang, J. Efficacy and safety of vonoprazan-based versus proton pump inhibitor-based triple therapy for Helicobacter pylori eradication: A meta-analysis of randomized clinical trials. BioMed Res. Int. 2019, 2019, 9781212-9781218. [CrossRef]

8. Floch, P.; Mégraud, F.; Lehours, P. Helicobacter pylori strains and gastric MALT lymphoma. Toxins 2017, 9, 132. [CrossRef]

9. Graham, D.Y.; Miftahussurur, M. Helicobacter pylori urease for diagnosis of Helicobacter pylori infection: A mini review. J. Adv. Res. 2018, 13, 51-57. [CrossRef]

10. Seta, T.; Takahashi, Y.; Noguchi, Y.; Shikata, S.; Sakai, T.; Sakai, K.; Yamashita, Y.; Nakayama, T. Effectiveness of Helicobacter pylori eradication in the prevention of primary gastric cancer in healthy asymptomatic 
people: A systematic review and meta-analysis comparing risk ratio with risk difference. PLoS ONE 2017, 12, e0183321. [CrossRef]

11. Ford, A.; Forman, D.; Hunt, R.; Yuan, Y.; Moayyedi, P. Helicobacter pylori eradication for the prevention of gastric neoplasia. Cochrane Database Syst. Rev. 2015. [CrossRef]

12. Suzuki, H.; Mori, H. World trends for H. pylori eradication therapy and gastric cancer prevention strategy by H. pylori test-and-treat. J. Gastroenterol. 2018, 53, 354-361. [CrossRef] [PubMed]

13. Scott, D.R.; Sachs, G.; Marcus, E.A. The role of acid inhibition in Helicobacter pylori eradication. F1000Research 2016, 5, 1747. [CrossRef] [PubMed]

14. Ierardi, E.; Losurdo, G.; La Fortezza, R.F.; Principi, M.; Barone, M.; Leo, A. Di Optimizing proton pump inhibitors in Helicobacter pylori treatment: Old and new tricks to improve effectiveness. World J. Gastroenterol. 2019, 25, 5097-5104. [CrossRef] [PubMed]

15. Putra, B.P.; Miftahussurur, M. Vonoprazan-based therapy has lower failure rate in eradicating Helicobacter pylori compared to proton pum inhibitors-based therapy: A meta-analysis of randomized controlled trials. New Armen. Med. J. 2019, 13, 22-30.

16. Graham, D.Y.; Dore, M.P. Update on the use of vonoprazan: A competitive acid blocker. Gastroenterology 2018, 154, 462-466. [CrossRef]

17. Malfertheiner, P.; Megraud, F.; O’Morain, C.; Gisbert, J.P.; Kuipers, E.J.; Axon, A.; Bazzoli, F.; Gasbarrini, A.; Atherton, J.; Graham, D.Y.; et al. Management of Helicobacter pylori infection-the Maastricht V/Florence consensus report. Gut 2017, 66, 6-30. [CrossRef]

18. Inatomi, N.; Matsukawa, J.; Sakurai, Y.; Otake, K. Potassium-competitive acid blockers: Advanced therapeutic option for acid-related diseases. Pharmacol. Ther. 2016, 168, 12-22. [CrossRef]

19. Rawla, P.; Sunkara, T.; Ofosu, A.; Gaduputi, V. Potassium-competitive acid blockers - are they the next generation of proton pump inhibitors? World J. Gastrointest. Pharmacol. Ther. 2018, 9, 63-68. [CrossRef]

20. Kato, M.; Ota, H.; Okuda, M.; Kikuchi, S.; Satoh, K.; Shimoyama, T.; Suzuki, H.; Handa, O.; Furuta, T.; Mabe, K.; et al. Guidelines for the management of Helicobacter pylori infection in Japan: 2016 Revised Edition. Helicobacter 2019, 24, e12597. [CrossRef]

21. Mori, H.; Suzuki, H. Role of acid suppression in acid-related diseases: Proton pump inhibitor and potassium-competitive acid blocker. J. Neurogastroenterol. Motil. 2019, 25, 6-14. [CrossRef] [PubMed]

22. Lee, K.J.; Son, B.K.; Kim, G.H.; Jung, H.K.; Jung, H.Y.; Chung, I.K.; Sung, I.K.; Kim, J.I.; Kim, J.H.; Lee, J.S.; et al. Randomised phase 3 trial: Tegoprazan, a novel potassium-competitive acid blocker, vs. esomeprazole in patients with erosive oesophagitis. Aliment. Pharmacol. Ther. 2019, 49, 864-872. [CrossRef] [PubMed]

23. Takahashi, N.; Take, Y. Tegoprazan, a novel potassium-competitive acid blocker to control gastric acid secretion and motility. J. Pharmacol. Exp. Ther. 2018, 364, 275-286. [CrossRef] [PubMed]

24. Iwakiri, K.; Kinoshita, Y.; Habu, Y.; Oshima, T.; Manabe, N.; Fujiwara, Y.; Nagahara, A.; Kawamura, O.; Iwakiri, R.; Ozawa, S.; et al. Evidence-based clinical practice guidelines for gastroesophageal reflux disease 2015. J. Gastroenterol. 2016, 51, 751-767. [CrossRef] [PubMed]

25. Chey, W.D.; Leontiadis, G.I.; Howden, C.W.; Moss, S.F. ACG clinical guideline: Treatment of Helicobacter pylori Infection. Am. J. Gastroenterol. 2017, 112, 212-238. [CrossRef]

26. Syam, A.F.; Simadibrata, M.; Makmun, D.; Abdullah, M.; Fauzi, A.; Renaldi, K.; Maulahela, H.; Utari, A.P. National consensus on management of dyspepsia and Helicobacter pylori infection. Acta Med. Indones. 2017, 49, 279-287.

27. Fallone, C.A.; Chiba, N.; van Zanten, S.V.; Fischbach, L.; Gisbert, J.P.; Hunt, R.H.; Jones, N.L.; Render, C.; Leontiadis, G.I.; Moayyedi, P.; et al. The Toronto consensus for the treatment of Helicobacter pylori infection in adults. Gastroenterology 2016, 151, 51-69.e4. [CrossRef]

28. Chang, J.Y.; Shim, K.N.; Tae, C.H.; Lee, K.E.; Lee, J.; Lee, K.H.; Moon, C.M.; Kim, S.E.; Jung, H.K.; Jung, S.A. Triple therapy versus sequential therapy for the first-line Helicobacter pylori eradication. BMC Gastroenterol. 2017, 17, 16. [CrossRef]

29. Thung, I.; Aramin, H.; Vavinskaya, V.; Gupta, S.; Park, J.Y.; Crowe, S.E.; Valasek, M.A. Review article: The global emergence of Helicobacter pylori antibiotic resistance. Aliment. Pharmacol. Ther. 2016, 43, 514-533. [CrossRef]

30. Miftahussurur, M.; Yamaoka, Y. Appropriate first-line regimens to combat Helicobacter pylori antibiotic resistance: An Asian perspective. Molecules 2015, 20, 6068-6092. [CrossRef] 
31. Miftahussurur, M.; Shrestha, P.K.; Subsomwong, P.; Sharma, R.P.; Yamaoka, Y. Emerging Helicobacter pylori levofloxacin resistance and novel genetic mutation in Nepal. BMC Microbiol. 2016, 16, 1-10. [CrossRef] [PubMed]

32. Shetty, V.; Lamichhane, B.; Tay, C.Y.; Pai, G.C.; Lingadakai, R.; Balaraju, G.; Shetty, S.; Ballal, M.; Chua, E.G. High primary resistance to metronidazole and levofloxacin, and a moderate resistance to clarithromycin in Helicobacter pylori isolated from Karnataka patients. Gut Pathog. 2019, 11, 21. [CrossRef] [PubMed]

33. Miftahussurur, M.; Waskito, L.A.; Syam, A.F.; Nusi, I.A.; Siregar, G.; Richardo, M.; Bakry, A.F.; Rezkitha, Y.A.A.; Wibawa, I.D.N.; Yamaoka, Y. Alternative eradication regimens for helicobacter pylori infection in indonesian regions with high metronidazole and levofloxacin resistance. Infect. Drug Resist. 2019, 12, 345-358. [CrossRef]

34. Echizen, $\mathrm{H}$. The first-in-class potassium-competitive acid blocker, vonoprazan fumarate: Pharmacokinetic and pharmacodynamic considerations. Clin. Pharmacokinet. 2016, 55, 409-418. [CrossRef]

35. Jenkins, H.; Sakurai, Y.; Nishimura, A.; Okamoto, H.; Hibberd, M.; Jenkins, R.; Yoneyama, T.; Ashida, K.; Ogama, Y.; Warrington, S. Randomised clinical trial: Safety, tolerability, pharmacokinetics and pharmacodynamics of repeated doses of TAK-438 (vonoprazan), a novel potassium-competitive acid blocker, in healthy male subjects. Aliment. Pharmacol. Ther. 2015, 41, 636-648. [CrossRef] [PubMed]

36. Sakurai, Y.; Nishimura, A.; Kennedy, G.; Hibberd, M.; Jenkins, R.; Okamoto, H.; Yoneyama, T.; Jenkins, H.; Ashida, K.; Irie, S.; et al. Safety, tolerability, pharmacokinetics, and pharmacodynamics of single rising Tak-438 (Vonoprazan) doses in healthy male Japanese/Non-Japanese Subjects. Clin. Transl. Gastroenterol. 2015, 6, e94. [CrossRef]

37. Shin, J.M.; Inatomi, N.; Munson, K.; Strugatsky, D.; Tokhtaeva, E.; Vagin, O.; Sachs, G. Characterization of a Novel Potassium-Competitive Acid Blocker of the Gastric H,K-ATPase, 1-[5-(2-Fluorophenyl)-1-(pyridin-3-ylsulfonyl)-1H-pyrrol-3-yl]-N-methylmethanamine Monofumarate (TAK-438). J. Pharmacol. Exp. Ther. 2011, 339, 412-420. [CrossRef]

38. Yang, X.; Li, Y.; Sun, Y.; Zhang, M.; Guo, C.; Mirza, I.A.; Li, Y.Q. Vonoprazan: A novel and potent alternative in the treatment of acid-related diseases. Dig. Dis. Sci. 2018, 63, 302-311. [CrossRef] [PubMed]

39. Wang, Y.; Wang, C.; Wang, S.; Zhou, Q.; Dai, D.; Shi, J.; Xu, X.; Luo, Q. Cytochrome P450-based drug-drug interactions of vonoprazan in vitro and in vivo. Front. Pharmacol. 2020, 11, 53. [CrossRef]

40. Jenkins, H.; Jenkins, R.; Patat, A. Effect of multiple oral doses of the potent CYP3A4 inhibitor clarithromycin on the pharmacokinetics of a single oral dose of vonoprazan: A phase I, open-label, sequential design study. Clin. Drug Investig. 2017, 37, 311-316. [CrossRef]

41. Kagami, T.; Sahara, S.; Ichikawa, H.; Uotani, T.; Yamade, M.; Sugimoto, M.; Hamaya, Y.; Iwaizumi, M.; Osawa, S.; Sugimoto, K.; et al. Potent acid inhibition by vonoprazan in comparison with esomeprazole, with reference to CYP2C19 genotype. Aliment. Pharmacol. Ther. 2016, 43, 1048-1059. [CrossRef] [PubMed]

42. Kinoshita, Y.; Ishimura, N.; Ishihara, S. Advantages and disadvantages of long-term proton pump inhibitor use. J. Neurogastroenterol. Motil. 2018, 24, 182-196. [CrossRef] [PubMed]

43. Oshima, T.; Arai, E.; Taki, M.; Kondo, T.; Tomita, T.; Fukui, H.; Watari, J.; Miwa, H. Randomised clinical trial: Vonoprazan versus lansoprazole for the initial relief of heartburn in patients with erosive oesophagitis. Aliment. Pharmacol. Ther. 2019, 49, 140-146. [CrossRef] [PubMed]

44. Oshima, T.; Miwa, H. Potent potassium-competitive acid blockers: A new era for the treatment of acid-related diseases. J. Neurogastroenterol. Motil. 2018, 24, 334-344. [CrossRef]

45. Akazawa, Y.; Fukuda, D.; Fukuda, Y. Vonoprazan-based therapy for Helicobacter pylori eradication: Experience and clinical evidence. Therap. Adv. Gastroenterol. 2016, 9, 845-852. [CrossRef]

46. Sugano, K. Vonoprazan fumarate, a novel potassium-competitive acid blocker, in the management of gastroesophageal reflux disease: Safety and clinical evidence to date. Therap. Adv. Gastroenterol. 2018, 11, 1-14. [CrossRef]

47. Yao, X.; Smolka, A.J. Gastric parietal cell physiology and Helicobacter pylori-induced disease. Gastroenterology 2019, 156, 2158-2173. [CrossRef]

48. Garnock-Jones, K.P. Vonoprazan: First global approval. Drugs 2015, 75, 439-443. [CrossRef]

49. Cheng, Y.; Liu, J.; Tan, X.; Dai, Y.; Xie, C.; Li, X.; Lu, Q.; Kou, F.; Jiang, H.; Li, J. Direct comparison of the efficacy and safety of vonoprazan versus proton-pump inhibitors for gastroesophageal reflux disease: A systematic review and meta-analysis. Dig. Dis. Sci. 2020. [CrossRef] 
50. Ashida, K.; Sakurai, Y.; Hori, T.; Kudou, K.; Nishimura, a.; Hiramatsu, N.; Umegaki, E.; Iwakiri, K. Randomised clinical trial: Vonoprazan, a novel potassium-competitive acid blocker, vs. lansoprazole for the healing of erosive oesophagitis. Aliment. Pharmacol. Ther. 2016, 43, 240-251. [CrossRef]

51. Mizokami, Y.; Oda, K.; Funao, N.; Nishimura, A.; Soen, S.; Kawai, T.; Ashida, K.; Sugano, K. Vonoprazan prevents ulcer recurrence during long-term NSAID therapy: Randomised, lansoprazole-controlled non-inferiority and single-blind extension study. Gut 2018, 67, 1042-1051. [CrossRef] [PubMed]

52. Miwa, H.; Uedo, N.; Watari, J.; Mori, Y.; Sakurai, Y.; Takanami, Y.; Nishimura, a.; Tatsumi, T.; Sakaki, N. Randomised clinical trial: Efficacy and safety of vonoprazan vs. lansoprazole in patients with gastric or duodenal ulcers-results from two phase 3, non-inferiority randomised controlled trials. Aliment. Pharmacol. Ther. 2017, 45, 240-252. [CrossRef] [PubMed]

53. Kawai, T.; Oda, K.; Funao, N.; Nishimura, A.; Matsumoto, Y.; Mizokami, Y.; Ashida, K.; Sugano, K. Vonoprazan prevents low-dose aspirin-associated ulcer recurrence: Randomised phase 3 study. Gut 2018, 67, 1033-1041. [CrossRef] [PubMed]

54. Jaruvongvanich, V.; Poonsombudlert, K.; Ungprasert, P. Vonoprazan versus proton-pump inhibitors for gastric endoscopic submucosal dissection-induced ulcers: A systematic review and meta-analysis. Eur. J. Gastroenterol. Hepatol. 2018, 30, 1416-1421. [CrossRef]

55. Sugimoto, M.; Yamaoka, Y. Role of vonoprazan in Helicobacter pylori eradication therapy in Japan. Front. Pharmacol. 2019, 9, 1-15. [CrossRef]

56. Waskito, L.A.; Miftahussurur, M.; Lusida, M.I.; Syam, A.F.; Suzuki, R.; Subsomwong, P.; Uchida, T.; Hamdan, M.; Nasronudin; Yamaoka, Y. Distribution and clinical associations of integrating conjugative elements and cag pathogenicity islands of Helicobacter pylori in Indonesia. Sci. Rep. 2018, 8, 6073. [CrossRef]

57. Doohan, D.; Miftahussurur, M.; Matsuo, Y.; Kido, Y.; Akada, J.; Matsuhisa, T.; Yee, T.T.; Htet, K.; Aftab, H.; Vilaichone, R.K.; et al. Characterization of a novel Helicobacter pylori East Asian-type CagA ELISA for detecting patients infected with various cagA genotypes. Med. Microbiol. Immunol. 2020, 209, $29-40$. [CrossRef]

58. Subsomwong, P.; Miftahussurur, M.; Uchida, T.; Vilaichone, R.K.; Ratanachu-Ek, T.; Mahachai, V.; Yamaoka, Y. Prevalence, risk factors, and virulence genes of Helicobacter pylori among dyspeptic patients in two different gastric cancer risk regions of Thailand. PLoS ONE 2017, 12, e0187113. [CrossRef]

59. Li, M.; Oshima, T.; Horikawa, T.; Tozawa, K.; Tomita, T.; Fukui, H.; Watari, J.; Miwa, H. Systematic review with meta-analysis: Vonoprazan, a potent acid blocker, is superior to proton-pump inhibitors for eradication of clarithromycin-resistant strains of Helicobacter pylori. Helicobacter 2018, 23, 1-8. [CrossRef]

60. Murakami, K.; Sakurai, Y.; Shiino, M.; Funao, N.; Nishimura, A.; Asaka, M. Vonoprazan, a novel potassium-competitive acid blocker, as a component of first-line and second-line triple therapy for Helicobacter pylori eradication: A phase III, randomised, double-blind study. Gut 2016, 65, 1439-1446. [CrossRef]

61. Maruyama, M.; Tanaka, N.; Kubota, D.; Miyajima, M.; Kimura, T.; Tokutake, K.; Imai, R.; Fujisawa, T.; Mori, H.; Matsuda, Y.; et al. Vonoprazan-based regimen is more useful than PPI-based one as a first-line Helicobacter pylori eradication: A randomized controlled trial. Can. J. Gastroenterol. Hepatol. 2017, 2017, 1-7. [CrossRef]

62. Sue, S.; Ogushi, M.; Arima, I.; Kuwashima, H.; Nakao, S.; Naito, M.; Komatsu, K.; Kaneko, H.; Tamura, T.; Sasaki, T.; et al. Vonoprazan- vs proton-pump inhibitor-based first-line 7-day triple therapy for clarithromycin-susceptible Helicobacter pylori: A multicenter, prospective, randomized trial. Helicobacter 2018, 23, e12456. [CrossRef]

63. Ozaki, H.; Harada, S.; Takeuchi, T.; Kawaguchi, S.; Takahashi, Y.; Kojima, Y.; Ota, K.; Hongo, Y.; Ashida, K.; Sakaguchi, M.; et al. Vonoprazan, a novel potassium-competitive acid blocker, should be used for the Helicobacter pylori eradication therapy as first choice: A large sample study of vonoprazan in real world compared with our randomized control trial using second-generation pro. Digestion 2018, 97, 212-218. [CrossRef]

64. Suzuki, S.; Gotoda, T.; Kusano, C.; Iwatsuka, K.; Moriyama, M. The efficacy and tolerability of a triple therapy containing a potassium-competitive acid blocker compared with a 7-day PPI-based low-dose clarithromycin triple therapy. Am. J. Gastroenterol. 2016, 111, 949-956. [CrossRef]

65. Shinozaki, S.; Nomoto, H.; Kondo, Y.; Sakamoto, H.; Hayashi, Y.; Yamamoto, H.; Lefor, A.K.; Osawa, H. Comparison of vonoprazan and proton pump inhibitors for eradication of Helicobacter pylori. Kaohsiung J. Med. Sci. 2016, 32, 255-260. [CrossRef] 
66. Shichijo, S.; Hirata, Y.; Niikura, R.; Hayakawa, Y.; Yamada, A.; Mochizuki, S.; Matsuo, K.; Isomura, Y.; Seto, M.; Suzuki, N.; et al. Vonoprazan versus conventional proton pump inhibitor-based triple therapy as first-line treatment against Helicobacter pylori: A multicenter retrospective study in clinical practice. J. Dig. Dis. 2016, 17, 670-675. [CrossRef]

67. Noda, H.; Noguchi, S.; Yoshimine, T.; Goji, S.; Adachi, K.; Tamura, Y.; Izawa, S.; Ebi, M.; Yamamoto, S.; Ogasawara, N.; et al. A novel potassium-competitive acid blocker improves the efficacy of clarithromycin-containing 7-day triple therapy against helicobacter pylori. J. Gastrointest. Liver Dis. 2016, 25, 283-288. [CrossRef]

68. Matsumoto, H.; Shiotani, A.; Katsumata, R.; Fujita, M.; Nakato, R.; Murao, T.; Ishii, M.; Kamada, T.; Haruma, K.; Graham, D.Y. Helicobacter pylori eradication with proton pump inhibitors or potassium-competitive acid blockers: The effect of clarithromycin resistance. Dig. Dis. Sci. 2016, 61, 3215-3220. [CrossRef]

69. Yamada, S.; Kawakami, T.; Nakatsugawa, Y.; Suzuki, T.; Fujii, H.; Tomatsuri, N.; Nakamura, H.; Sato, H.; Okuyama, Y.; Kimura, H.; et al. Usefulness of vonoprazan, a potassium ion-competitive acid blocker, for primary eradication of Helicobacter pylori. World J. Gastrointest. Pharmacol. Ther. 2016, 7, 550. [CrossRef]

70. Tsujimae, M.; Yamashita, H.; Hashimura, H.; Kano, C.; Shimoyama, K.; Kanamori, A.; Matsumoto, K.; Koizumi, A.; Momose, K.; Eguchi, T.; et al. A comparative study of a new class of gastric acid suppressant agent named vonoparazan versus esomeprazole for the eradication of Helicobacter pylori. Digestion 2017, 94, 240-246. [CrossRef]

71. Kajihara, Y.; Shimoyama, T.; Mizuki, I. Analysis of the cost-effectiveness of using vonoprazan-amoxicillin-clarithromycin triple therapy for first-line Helicobacter pylori eradication. Scand. J. Gastroenterol. 2017, 52, 238-241. [CrossRef]

72. Sakurai, K.; Suda, H.; Ido, Y.; Takeichi, T.; Okuda, A.; Hasuda, K.; Hattori, M. Comparative study: Vonoprazan and proton pump inhibitors in Helicobacter pylori eradication therapy. World J. Gastroenterol. 2017, 23, 668-675. [CrossRef]

73. Sue, S.; Kuwashima, H.; Iwata, Y.; Oka, H.; Arima, I.; Fukuchi, T.; Sanga, K.; Inokuchi, Y.; Ishii, Y.; Kanno, M.; et al. The superiority of vonoprazan-based first-line triple therapy with clarithromycin: A prospective multi-center cohort study on Helicobacter pylori eradication. Intern. Med. 2017, 56, 1277-1285. [CrossRef]

74. Nishizawa, T.; Suzuki, H.; Hibi, T. Quinolone-based therapy for Helicobacter pylori eradication. J. Clin. Biochem. Nutr. 2009, 44, 119-124. [CrossRef]

75. Tanabe, H.; Yoshino, K.; Ando, K.; Nomura, Y.; Ohta, K.; Satoh, K.; Ichiishi, E.; Ishizuka, A.; Otake, T.; Kohgo, Y.; et al. Vonoprazan-based triple therapy is non-inferior to susceptibility-guided proton pump inhibitor-based triple therapy for Helicobacter pylori eradication. Ann. Clin. Microbiol. Antimicrob. 2018, 17, 1-7. [CrossRef]

76. Shinozaki, S.; Shinozaki, S.; Kobayashi, Y.; Osawa, H.; Sakamoto, H.; Hayashi, Y.; Yamamoto, H.; Lefor, A.K. Effectiveness and safety of vonoprazan versus proton pump inhibitors for second-line Helicobacter pylori eradication therapy: Systematic review and meta-analysis. Digestion 2020, 3223, 1-7. [CrossRef]

77. Nishizawa, T.; Suzuki, H.; Fujimoto, A.; Kinoshita, H.; Yoshida, S.; Isomura, Y.; Toyoshima, A.; Kanai, T.; Yahagi, N.; Toyoshima, O. Effects of patient age and choice of antisecretory agent on success of eradication therapy for Helicobacter pylori infection. J. Clin. Biochem. Nutr. 2017, 60, 208-210. [CrossRef]

78. Sue, S.; Shibata, W.; Sasaki, T.; Kaneko, H.; Irie, K.; Kondo, M.; Maeda, S. Randomized trial of vonoprazan-based versus proton-pump inhibitor-based third-line triple therapy with sitafloxacin for Helicobacter pylori. J. Gastroenterol. Hepatol. 2019, 34, 686-692. [CrossRef]

79. Saito, Y.; Konno, K.; Sato, M.; Nakano, M.; Kato, Y.; Saito, H.; Serizawa, H. Vonoprazan-based third-line therapy has a higher eradication rate against sitafloxacin-resistant. Cancers 2019, 11, 116. [CrossRef]

80. Kiyotoki, S.; Nishikawa, J.; Sakaida, I. Efficacy of vonoprazan for helicobacter pylori eradication. Intern. Med. 2020, 59, 153-161. [CrossRef]

81. Okamura, T.; Suga, T.; Nagaya, T.; Arakura, N.; Matsumoto, T.; Nakayama, Y.; Tanaka, E. Antimicrobial resistance and characteristics of eradication therapy of Helicobacter pylori in Japan: A multi-generational comparison. Helicobacter 2014, 19, 214-220. [CrossRef]

82. Miftahussurur, M.; Aftab, H.; Shrestha, P.K.; Sharma, R.P.; Subsomwong, P.; Waskito, L.A.; Doohan, D.; Fauzia, K.A.; Yamaoka, Y. Effective therapeutic regimens in two South Asian countries with high resistance to major Helicobacter pylori antibiotics. Antimicrob. Resist. Infect. Control. 2019, 8, 1-10. [CrossRef] 
83. Chaudhary, P.H.; Tawar, M.G. Pharmacognostic and phytopharmacological overview on Bombax ceiba. Syst. Rev. Pharm. 2019, 10, 20-25. [CrossRef]

84. Ratnasari, N.; Rezkitha, Y.A.A.; Adnyana, I.K.; Alfaray, R.I.; Fauzia, K.A.; Doohan, D.; Panjaitan, A.; Priskila, Y.; Yulinah, E.; Khomsan, A.; et al. Anti-helicobacter pylori effects of propolis ethanol extract on clarithromycin and metronidazole resistant strains. Syst. Rev. Pharm. 2020, 11, 429-434. [CrossRef]

85. Scott, D.R.; Munson, K.B.; Marcus, E.A.; Lambrecht, N.W.G.; Sachs, G. The binding selectivity of vonoprazan (TAK-438) to the gastric H+,K+-ATPase. Aliment. Pharmacol. Ther. 2015, 42, 1315-1326. [CrossRef] [PubMed]

86. Lundell, L.; Vieth, M.; Gibson, F.; Nagy, P.; Kahrilas, P.J. Systematic review: The effects of long-term proton pump inhibitor use on serum gastrin levels and gastric histology. Aliment. Pharmacol. Ther. 2015, 42, 649-663. [CrossRef] [PubMed]

87. Sundaresan, S.; Kang, A.J.; Merchant, J.L. Pathophysiology of gastric NETs: Role of gastrin and menin. Curr. Gastroenterol. Rep. 2017, 19, 1-12. [CrossRef]

88. Martinsen, T.C.; Fossmark, R.; Waldum, H.L. The phylogeny and biological function of gastric juice-Microbiological consequences of removing gastric acid. Int. J. Mol. Sci. 2019, 20, 6031. [CrossRef]

89. Bruno, G.; Zaccari, P.; Rocco, G.; Scalese, G.; Panetta, C.; Porowska, B.; Pontone, S.; Severi, C. Proton pump inhibitors and dysbiosis: Current knowledge and aspects to be clarified. World J. Gastroenterol. 2019, 25, 2706-2719. [CrossRef]

90. Heidelbaugh, J.J. Proton pump inhibitors and risk of vitamin and mineral deficiency: Evidence and clinical implications. Ther. Adv. Drug Saf. 2013, 4, 125-133. [CrossRef]

91. Kristanto, A.; Adiwinata, R.; Rasidi, J.; Phang, B.B.; Adiwinata, S.; Richard, T.; Uwan, W.B.; Syam, A.F. Long-term risks of proton pump inhibitor administration: A literature review. Indones. J. Gastroenterol. Hepatol. Dig. Endosc. 2017, 18, 169-176. [CrossRef]

92. Maes, M.L.; Fixen, D.R.; Linnebur, S.A. Adverse effects of proton-pump inhibitor use in older adults: A review of the evidence. Ther. Adv. Drug Saf. 2017, 8, 273-297. [CrossRef] [PubMed]

93. Hussain, S.; Singh, A.; Habib, A.; Najmi, A.K. Proton pump inhibitors use and risk of chronic kidney disease: Evidence-based meta-analysis of observational studies. Clin. Epidemiol. Glob. Health 2019, 7, 46-52. [CrossRef]

(C) 2020 by the authors. Licensee MDPI, Basel, Switzerland. This article is an open access article distributed under the terms and conditions of the Creative Commons Attribution (CC BY) license (http://creativecommons.org/licenses/by/4.0/). 